\title{
Multivariate spectral multipliers for tensor product orthogonal expansions
}

\author{
Błażej Wróbel
}

Received: 1 April 2011 / Accepted: 11 November 2011 / Published online: 29 November 2011

C The Author(s) 2011. This article is published with open access at Springerlink.com

\begin{abstract}
By using a multi-dimensional analogue of the Mellin transform techniques we prove a multivariate multiplier theorem for general tensor product orthogonal expansions and a multivariate multiplier theorem for the Hankel transform.
\end{abstract}

Keywords Multivariate spectral multipliers · Mellin transform ·

Orthogonal expansions

Mathematics Subject Classification (2010) Primary 42B15; Secondary 42C10

\section{Introduction}

In this paper we develop a multi-dimensional analogue of the Mellin transform techniques from Meda's paper [13] in order to obtain a multivariate multiplier theorem for general tensor product orthogonal expansions. Without much more effort, the methods presented in our paper lead to more general results; stated in the context of joint spectral multipliers of operators with positive, not necessarily discrete spectra, acting on separate variables. However, we feel that in the context of orthogonal expansions the results are clearer and still retain much of their applicable generality. The setting of operators with non-negative spectra acting on separate variables has been investigated

Communicated by K. Gröchenig.

The research was supported by Polish Government funds for science-research project 2011/01/N/ST1/01785, NCN.

B. Wróbel (凶)

Institute of Mathematics of University of Wrocław, pl. Grunwaldzki 2/4, 50-384 Wrocław, Poland e-mail: blazej.wrobel@math.uni.wroc.pl 
by Sikora in [18]. What distinguishes our paper from the latter is the fact that in a relevant place (see Sect. 4) we assume only some polynomial bounds on the $L^{p}$ norms of the imaginary power operators. These hold if the heat semigroups corresponding to the operators in question possess Gaussian bounds, which are assumed to hold in [18]. However, there are some cases when the norms of the imaginary power operators have bounds appropriate for the present paper but the corresponding heat semigroup does not have Gaussian bounds. This is the case of the Jacobi expansions, see Proposition 4.2. Moreover, the Marcinkiewicz condition of our paper, see (4.2), has a different form than the one implied by the assumptions made in [18, Theorem 2.1]. In fact, to some extent, we answer the question posed by Sikora in [18, Remark 4].

We may also consider joint spectral multipliers for more general system of strongly commuting operators, not necessarily acting on separate variables. For a treatise of the subject in the context of general Lie groups of polynomial growth see [11] and the references given therein. Marcinkiewicz-type results on joint spectral multipliers for specific commuting operators (i.e sublaplacians and central derivatives) on the Heisenberg (and related) groups, were known earlier, see [6-8, 12,14, 15,24]. Note that in all the papers already mentioned in this paragraph the Marcinkiewicz condition assumed is similar to ours, while the smoothness threshold needed is sharper. In fact, in most of those papers the smoothness threshold assumed is 'sharp', while in our Theorem 4.1 one order of smoothness is lost. For results on holomorphic functional calculi for joint spectral multipliers see for instance [1].

The theorem we are aiming to generalize is [13, Theorem 1]. Its proof appears in a simpler form also in [3], however the complex variable techniques used in the latter paper seem to be inapplicable in our case. The main idea of our paper may be outlined as follows. Since a nice function $m:(0, \infty)^{d} \rightarrow \mathbb{C}$ may be expressed as an integral of its Mellin transform times the product of the imaginary powers $\lambda_{n}^{i u_{n}}, n=1, \ldots, d$, see (2.7), we expect something similar to hold for the multivariate spectral multiplier operator for some tensor product orthogonal expansions (with the imaginary powers replaced by the imaginary power operators).

It should be noted that mostly, the general framework we work in does not allow to obtain 'sharp' results. For instance, the smoothness requirements in Theorem 4.1 can be often lowered e.g., when the space in question possesses some dilation/translation structure. That is the case when working on Lie groups (see [11] and references given therein) but also in the case of the Hankel transform, for which we sketch a proof of a 'sharp' multivariate multiplier theorem in the Appendix.

With the exceptions of Sect. 5 and Appendix, throughout the paper we use the assumption that 0 is in the resolvent set of all the operators we consider. The main reason for this restriction is that the imaginary powers of 0 are not defined. In the one dimensional case this is not a very serious obstacle, and it has been overcome for instance for the Ornstein-Uhlenbeck operator, see [9]. In the multi-dimensional case this will be one of the issues of the forthcoming paper by the author.

The paper is organized as follows. Section 2 contains the setup and the statements of the general Theorems 2.1 and 2.2. Theorem 2.1 which is the most direct result is justified already in Sect. 2. In order to prove Theorem 2.2, which can be thought of as the main result of the paper we need to develop some multi-parameter $g$-function 
techniques, see Theorem 2.4. The proofs of Theorems 2.2 and 2.4 are given in Sect. 3. Even though in the proof of Theorem 2.2 we follow fairly closely the proof of [13, Theorem 1], the main tools we use to prove the prerequisite Theorem 2.4 are different and consist of the inversion formula and the Plancherel formula for the Mellin transform. The first paragraph of Sect. 4 is devoted to proving a multiplier theorem in the case of polynomial growth of the norms of the imaginary power operators, see Theorem 4.1. This is achieved in the spirit of [13, Theorem 4]. The polynomial growth condition for the norms of the imaginary power operators is satisfied for instance by invariant Laplacians or sublaplacians on groups of polynomial growth, or by Laplace-Beltrami operators on compact manifolds. In the rest of Sect. 4 we give some applications of Theorem 4.1. One of the illustrations is a multivariate multiplier theorem for the Jacobi expansions, 'en route' to prove which we obtain some bounds for the norms of the imaginary power operators connected with the Jacobi operator, see Proposition 4.2. In Sect. 5 we demonstrate how to use the techniques of the paper to obtain a multivariate multiplier theorem for the Hankel transform, which may be thought of as a continuous multi-dimensional orthogonal system, where the 'basis' is a tensor product. In Appendix we briefly show how to lower the smoothness assumptions in the case of the Hankel transform by exploiting dilation/translation structure connected with this setting.

We use the following notation. The symbol $L^{p}, 1<p<\infty$ will denote (if not stated otherwise) the space $L^{p}(A, v)=L^{p}\left(A_{1} \times \cdots \times A_{d}, v_{1} \otimes \cdots \otimes v_{d}\right)$, with the norm $\|\cdot\|_{p}$. By $\|\cdot\|_{p \rightarrow p}$ we mean the operator norm from either $L^{p}$ to $L^{p}$ or from $L^{p}\left(A_{n}, v_{n}\right)$ to $L^{p}\left(A_{n}, v_{n}\right)$ (it should be clear from the context to which space we are referring to). By $\mathbb{N}$ we mean the set of nonnegative integers. The symbol $\langle\cdot, \cdot\rangle$ will denote the inner product either in the space $L^{2}(A, v)$ or in $L^{2}\left(A_{n}, v_{n}\right)$. We will also write $f \sim \sum_{k \in \mathbb{N}} a_{k} e_{k}$ to denote that $f$ has the expansion $\sum_{k \in \mathbb{N}} a_{k} e_{k}$ in some basis $e_{k}$ of $L^{2}$. The notation $X \lesssim Y$ will be used to indicate that $X \leq C Y$ with a positive constant $C$ independent of significant quantities. For vectors $\mathbf{X}, \mathbf{Y}$ (in particular for multi-indices) we will write $\mathbf{X}<\mathbf{Y}$ whenever $X_{n}<Y_{n}, \ldots, n=1, \ldots, d$. The symbol 1 will denote the vector $(1, \ldots, 1)$.

\section{Preliminaries and some basic results}

Let $\left\{e_{k}\right\}_{k \in \mathbb{N}^{d}}, e_{k}=e_{k_{1}}^{1} \times \cdots \times e_{k_{d}}^{d}$ be an orthonormal basis of the space $(A, v)$, with $A=A_{1} \times \cdots \times A_{d}, d v(x)=d \nu_{1}\left(x_{1}\right) \ldots d v_{d}\left(x_{d}\right)$, and $k=\left(k_{1}, \ldots, k_{d}\right)$ being a multiindex. The number $d$ will be called the dimension. However, the reader should be aware that the spaces $A_{n}$ themselves might also be $l_{n}$-dimensional in some sense, whereas each of the system $\left\{e_{r}^{n}\right\}_{r=0, \ldots}$, might already be of the tensor product form. While considering the expansions of Hermite type we may take for instance $A_{n}=\mathbb{R}^{l_{n}}$ and $e_{r}^{n}$ to be the $l_{n}$-dimensional Hermite functions.

Assume that for each $n=1, \ldots, d,\left\{e_{r}^{n}\right\}_{r=0,1, \ldots}$ is an eigenfunction decomposition of some self-adjoint, positive operator $\mathcal{L}_{n}$ in $\left(L^{2}\left(A_{n}\right), v_{n}\right)$ with strictly positive eigenvalues $0<\lambda_{0}^{n}<\lambda_{1}^{n}<\cdots$, i.e.

$$
\mathcal{L}_{n} f=\sum_{r=0}^{\infty} \lambda_{r}^{n}\left\langle f, e_{r}^{n}\right\rangle e_{r}^{n}
$$


on the domain

$$
\operatorname{Dom}\left(\mathcal{L}_{n}\right)=\left\{f \in L^{2}\left(A_{n}, v_{n}\right): \sum_{r=0}^{\infty}\left(\lambda_{r}^{n}\right)^{2}\left|\left\langle f, e_{r}^{n}\right\rangle\right|^{2}<\infty\right\} .
$$

Let $T_{t_{n}}^{n}$ be the heat semigroup corresponding to $\mathcal{L}_{n}$, that is

$$
T_{t_{n}}^{n} f=e^{-t_{n} \mathcal{L}_{n}} f=\sum_{r=0}^{\infty} e^{-t_{n} \lambda_{r}^{n}}\left\langle f, e_{r}^{n}\right\rangle e_{r}^{n}, \quad f \in L^{2}\left(A_{n}, v_{n}\right)
$$

For a bounded function $m:(0, \infty)^{d} \rightarrow \mathbb{C}$ define the operator

$$
m(\mathcal{L}) f=m\left(\mathcal{L}_{1}, \ldots, \mathcal{L}_{d}\right) f=\sum_{k \in \mathbb{N}^{d}} m\left(\lambda_{k_{1}}^{1}, \ldots, \lambda_{k_{d}}^{d}\right)\left\langle f, e_{k}\right\rangle e_{k}, \quad f \in L^{2}
$$

The multiplier $m$ can be also viewed as a joint spectral multiplier of the operators $\mathcal{L}_{n}, n=1, \ldots, d$ acting on separate variables. Obviously $m(\mathcal{L})$ is bounded on $L^{2}$. It is also easy to observe that for two bounded functions $m_{1}, m_{2}$, we have

$$
m_{1}(\mathcal{L}) m_{2}(\mathcal{L})=\left(m_{1} m_{2}\right)(\mathcal{L})=m_{2}(\mathcal{L}) m_{1}(\mathcal{L}) .
$$

In particular, taking $m\left(\lambda_{k_{1}}^{1}, \ldots, \lambda_{k_{d}}^{d}\right)=\left(\lambda_{k_{1}}^{1}\right)^{i u_{1}} \times \cdots \times\left(\lambda_{k_{d}}^{d}\right)^{i u_{d}}$, we obtain

$$
\mathcal{L}^{i u_{1}, \ldots, i u_{d}} f=\sum_{k \in \mathbb{N}^{d}}\left(\lambda_{k_{1}}^{1}\right)^{i u_{1}} \times \cdots \times\left(\lambda_{k_{d}}^{d}\right)^{i u_{d}}\left\langle f, e_{k}\right\rangle e_{k},
$$

with $u_{n} \in \mathbb{R}, n=1, \ldots, d$. Define the imaginary powers of the operators $\mathcal{L}_{n}^{i u_{n}}$ as

$$
\mathcal{L}_{n}^{i u_{n}} f=\sum_{r=0}^{\infty}\left(\lambda_{r}^{n}\right)^{i u_{n}}\left\langle f_{n}, e_{r}^{n}\right\rangle e_{r}^{n}, \quad f_{n} \in L^{2}\left(A_{n}, d v_{n}\right), \quad n=1, \ldots, d .
$$

The above can be also understood as the operators of the form (2.3) (with $u_{i}=0$ for $i \neq n$ ) acting on the space $L^{2}$. In dimension $d=2$ it can be easily noted that

$$
\mathcal{L}^{i u_{1}, i u_{2}} f=\mathcal{L}_{1}^{i u_{1}} \mathcal{L}_{2}^{i u_{2}} f=\mathcal{L}_{2}^{i u_{2}} \mathcal{L}_{1}^{i u_{1}} f, \quad f \in L^{2} .
$$

Also for $f \in L^{p} \cap L^{2}, 1<p<\infty$,

$$
\begin{aligned}
\left\|\mathcal{L}_{1}^{i u_{1}} \mathcal{L}_{2}^{i u_{2}}(f)\right\|_{p}^{p} & \leq \int_{A_{2}}\left\|\mathcal{L}_{1}^{i u_{1}}\right\|_{p \rightarrow p}^{p}\left(\int_{A_{1}}\left|\mathcal{L}_{2}^{i u_{2}}(f)\right|^{p} d v_{1}(x)\right) d v_{2}(y) \\
& \leq\left\|\mathcal{L}_{1}^{i u_{1}}\right\|_{p \rightarrow p}^{p}\left\|\mathcal{L}_{2}^{i u_{2}}\right\|_{p \rightarrow p}^{p}\|f\|_{p}^{p},
\end{aligned}
$$


so that the operator $\mathcal{L}^{i u_{1}, i u_{2}}$ has a bounded extension onto $L^{p}$, which satisfies

$$
\left\|\mathcal{L}^{i u_{1}, i u_{2}}\right\|_{p \rightarrow p} \leq\left\|\mathcal{L}_{1}^{i u_{1}}\right\|_{p \rightarrow p}\left\|\mathcal{L}_{2}^{i u_{2}}\right\|_{p \rightarrow p}
$$

(provided the right hand side of the above is finite). The above reasoning can be easily generalized to higher dimensions, producing

$$
\left\|\mathcal{L}^{i u_{1}, \ldots, i u_{d}}\right\|_{p \rightarrow p} \leq \prod_{n=1}^{d}\left\|\mathcal{L}_{n}^{i u_{n}}\right\|_{p \rightarrow p}
$$

Note that on the right hand side of the above inequality the symbol $\|\cdot\|_{p \rightarrow p}$ denotes the operator norms on the spaces $L^{p}\left(A_{n}, v_{n}\right), n=1, \ldots, d$. Inequality (2.6) is one of the reasons for the restriction to orthogonal expansions of the tensor product form. We could define multivariate multiplier operators of the form (2.1), and generalized imaginary power operators of the form (2.3) for other orthogonal expansions and then try to apply Mellin transform techniques. However, we would not be able to obtain an estimate of the form (2.6), thus we could not say much about the $L^{p}$ norms of the operators of the form (2.3).

For a function $m \in L^{1}\left((0, \infty)^{d}, \frac{d \lambda}{\lambda}\right)$, where $\frac{d \lambda}{\lambda} \equiv \frac{d \lambda_{1}}{\lambda_{1}} \cdots \frac{d \lambda_{d}}{\lambda_{d}}$, define its $d$-dimensional Mellin transform by

$$
\mathcal{M}(m)(u)=\int_{(0, \infty)^{d}} \prod_{n=1}^{d} \lambda_{n}^{-i u_{n}} m\left(\lambda_{1}, \ldots, \lambda_{d}\right) \frac{d \lambda}{\lambda},
$$

where the vector $u=\left(u_{1}, \ldots, u_{d}\right) \in \mathbb{R}^{d}$. In the dimension $d=2$, assuming $\mathcal{M}(m) \in$ $L^{1}\left(\mathbb{R}^{2}, d u\right)$, the following inversion formula holds

$$
m(\xi, \eta)=C \int_{\mathbb{R}^{2}} \xi^{i u_{1}} \eta^{i u_{2}} \mathcal{M}(m)\left(u_{1}, u_{2}\right) d u .
$$

Note that the assumptions that $m$ and $\mathcal{M}(m)$ belong to the appropriate $L^{1}$ spaces imply that they are both continuous and the inversion formula (2.7) holds for every $\xi, \eta$. We also have a Plancherel formula in this context, namely,

$$
\int_{0}^{\infty} \int_{0}^{\infty}|m(\xi, \eta)|^{2} \frac{d \xi}{\xi} \frac{d \eta}{\eta}=\left(4 \pi^{2}\right)^{-1} \int_{\mathbb{R}^{2}}\left|\mathcal{M}(m)\left(u_{1}, u_{2}\right)\right|^{2} d u
$$

where the extension of $\mathcal{M}$ from $L^{1}\left((0, \infty)^{2}, \frac{d \xi}{\xi} \frac{d \eta}{\eta}\right)$ to $L^{2}\left((0, \infty)^{2}, \frac{d \xi}{\xi} \frac{d \eta}{\eta}\right)$ is made exactly as in the case of Fourier transform on $\mathbb{R}^{2}$ (observe that for the change of variable $z=\log (\xi), w=\log (\eta)$, we have $d z=\frac{d \xi}{\xi}, d w=\frac{d \eta}{\eta}$,). Results analogous to (2.7) and (2.8) hold also for dimensions $d>2$.

Our first theorem is the following. 
Theorem 2.1 Let $m$ be a bounded function on $(0, \infty)^{d}$ satisfying the assumptions

(i) $m \in L^{1}\left((0, \infty)^{d}, \frac{d \lambda}{\lambda}\right)$,

(ii) $\int_{\mathbb{R}^{d}}\left|\mathcal{M}(m)\left(u_{1}, \ldots, u_{d}\right)\right|\left(\left\|\mathcal{L}^{i u_{1}, \ldots, i u_{d}}\right\|_{p \rightarrow p}+1\right) d u<\infty$,

for some $p \in(1, \infty)$. Then the operator $m(\mathcal{L})$ defined on $L^{2}$ by $(2.1)$ extends to a bounded operator on $L^{p}$.

Proof We will prove the theorem in the dimension $d=2$. In higher dimensions the proof is analogous. From the assumptions the inversion formula (2.7) holds, hence it can be easily checked that for say, $f \in L^{2} \cap L^{p}$,

$$
m(\mathcal{L}) f=\left(4 \pi^{2}\right)^{-1} \int_{\mathbb{R}^{2}} \mathcal{M}(m)\left(u_{1}, u_{2}\right) \mathcal{L}^{i u_{1}, i u_{2}} f d u,
$$

where the right hand side is understood as a Bochner integral taking values in $L^{2}$. Applying Minkowski's integral inequality we deduce that

$$
\|m(\mathcal{L}) f\|_{p} \leq\left(4 \pi^{2}\right)^{-1} \int_{\mathbb{R}^{2}}\left|\mathcal{M}(m)\left(u_{1}, u_{2}\right)\right|\left\|\mathcal{L}^{i u_{1}, i u_{2}}\right\|_{p \rightarrow p} d u\|f\|_{p} \lesssim\|f\|_{p} .
$$

For our main theorem we need some more notation. For $N \in \mathbb{N}, T=\left(t_{1}, \ldots, t_{d}\right) \in$ $(0, \infty)^{d}$ and $\lambda=\left(\lambda_{1}, \ldots, \lambda_{d}\right) \in(0, \infty)^{d}$ let

$$
m_{N, T}(\lambda)=\prod_{n=1}^{d}\left(t_{n} \lambda_{n}\right)^{N} \exp \left(-\sum_{n=1}^{d} t_{n} \lambda_{n}\right) m(\lambda)
$$

The main theorem we prove is a multivariate analogue of [13, Theorem 1].

Theorem 2.2 Assume that for some $1<p<\infty$, some positive integer $N$ and nonnegative integer $M$ the following conditions hold

(i)

$$
\int_{\mathbb{R}^{d}} \sup _{T \in(0, \infty)^{d}}\left|\mathcal{M}\left(m_{N, T}\right)\left(u_{1}, \ldots, u_{d}\right)\right|\left\|\mathcal{L}^{i u_{1}, \ldots, i u_{d}}\right\|_{p \rightarrow p} d u<\infty
$$

(ii)

$$
\max _{n=1, \ldots, d}\left(\left\|\mathcal{L}_{n}^{i v}\right\|_{L^{p}\left(A_{n}, v_{n}\right)}\right) \lesssim(1+|v|)^{M} \exp (\pi|1 / p-1 / 2||v|), \quad v \in \mathbb{R} .
$$

Then $m(\mathcal{L})$ extends to a bounded operator on $L^{p}$. 
Note that from [2] we know that if the semigroups $\left\{T_{\tau}^{n}\right\}_{\tau>0}, n=1, \ldots, d$ are semigroups of contractions on all $L^{p}\left(A_{n}, v_{n}\right)$ spaces, $1 \leq p \leq \infty$, then (2.11) is satisfied with $M=4|1 / p-1 / 2|$. We will justify the above theorem in the next section and for $d=2$, in higher dimensions the reasoning is analogous. The proof of the theorem relies on the $L^{p}$ boundedness of some auxiliary $g$-function $g_{N}$. Let

$$
g_{N}(f)\left(x_{1}, x_{2}\right)=\left(\int_{0}^{\infty} \int_{0}^{\infty}\left|t^{N} s^{N}\left(\frac{d^{N}}{d t^{N}} T_{t}^{1} \frac{d^{N}}{d s^{N}} T_{s}^{2} f\right)\left(x_{1}, x_{2}\right)\right|^{2} \frac{d t}{t} \frac{d s}{s}\right)^{1 / 2} .
$$

Here and later on the expression $\frac{d^{N}}{d t^{N}} T_{t}^{1} \frac{d^{N}}{d s^{N}} T_{s}^{2} f$ is understood as an iteration of the one-dimensional $L^{2}\left(A_{n}, v_{n}\right)$ derivative operators $\frac{d^{N}}{d \tau^{N}} T_{\tau}^{n}, n=1,2, \tau=t$, s, which act on $f \in L^{2}$ with the expansion $\sum_{k \in \mathbb{N}^{2}} a_{k} e_{k}$, as

$$
\frac{d^{N}}{d \tau^{N}} T_{\tau}^{n} f=(-1)^{N} \sum_{k \in \mathbb{N}^{2}}\left(\lambda_{k_{n}}^{n}\right)^{N} e^{-\tau \lambda_{k_{n}}^{n}} a_{k} e_{k}, \quad n=1,2, \quad \tau=t, s .
$$

It is obvious that these operators commute on $L^{2}$ and

$$
\frac{d^{N}}{d t^{N}} T_{t}^{1} \frac{d^{N}}{d s^{N}} T_{s}^{2} f=\sum_{k \in \mathbb{N}^{2}}\left(\lambda_{k_{1}}^{1} \lambda_{k_{2}}^{2}\right)^{N} e^{-\lambda_{k_{1}}^{1} t} e^{-\lambda_{k_{2}}^{2} s} a_{k} e_{k}
$$

In many concrete cases, when the semigroups $T_{\tau}^{n}$ have integral kernels $G_{\tau}^{n}\left(x_{n}, y_{n}\right)$ we could instead consider pointwise differentiation, i.e.

$$
\frac{d^{N}}{d \tau^{N}} T_{\tau}^{n} f\left(x_{n}\right)=\int_{A_{n}} \frac{d^{N}}{d \tau^{N}} G_{\tau}^{n}\left(x_{n}, y_{n}\right) f\left(y_{n}\right) d v_{n}\left(y_{n}\right), \quad n=1,2, \quad \tau=t, s
$$

and (notation: $\left.G_{t, s}(x, y)=G_{t}^{1}\left(x_{1}, y_{1}\right) G_{s}^{2}\left(x_{2}, y_{2}\right)\right)$

$$
\frac{d^{N}}{d t^{N}} T_{t}^{1} \frac{d^{N}}{d s^{N}} T_{s}^{2} f(x)=\int_{A} \frac{d^{N}}{d t^{N}} \frac{d^{N}}{d s^{N}} G_{t, s}(x, y) d v(y) .
$$

First we observe the following.

Proposition $2.3 g_{N}$ is an isometry on $L^{2}$, i.e $\left\|g_{N}(f)\right\|_{2}=\|f\|_{2}$.

Proof Using Fubini's theorem several times together with the fact that the one-dimensional $g$-functions $g_{N}^{n}$, defined by

$$
\left(g_{N}^{n}\left(f_{n}\right)\right)^{2}=\int_{0}^{\infty}\left|\tau^{N}\left(\frac{d^{N}}{d \tau^{N}} T_{\tau}^{n}\right)\left(f_{n}\right)\right|^{2} \frac{d \tau}{\tau}, \quad f_{n} \in L^{2}\left(A_{n}, v_{n}\right), \quad n=1,2
$$


can be easily seen to preserve $L^{2}\left(A_{n}, v_{n}\right)$ norms, we write

$$
\begin{aligned}
& \int_{A_{2}} \int_{A_{1}}\left(g_{N}(f)\left(x_{1}, x_{2}\right)\right)^{2} d v_{1}\left(x_{1}\right) d v_{2}\left(x_{2}\right) \\
& =\int_{A_{2}} \int_{0}^{\infty}\left(\int_{A_{1}} \int_{0}^{\infty}\left|t^{N} \frac{d^{N}}{d t^{N}} T_{t}^{1}\left(\frac{d^{N}}{d s^{N}} T_{s}^{2} f\right)\left(x_{1}, x_{2}\right)\right|^{2} \frac{d t}{t} d v_{1}\left(x_{1}\right)\right) s^{2 N-1} d s d \nu_{2}\left(x_{2}\right) \\
& =\int_{A_{1}}\left(\int_{A_{2}} \int_{0}^{\infty}\left|\left(s^{N} \frac{d^{N}}{d s^{N}} T_{s}^{2} f\right)\left(x_{1}, x_{2}\right)\right|^{2} \frac{d s}{s} d v_{2}\left(x_{2}\right)\right) d v_{1}\left(x_{1}\right) \\
& =\int_{A_{1}} \int_{A_{2}}\left|f\left(x_{1}, x_{2}\right)\right|^{2} d v_{1}\left(x_{1}\right) d v_{2}\left(x_{2}\right)=\|f\|_{2}^{2} .
\end{aligned}
$$

In particular from the above it follows that $g_{N}(f)$ is well defined $\left(x_{1}, x_{2}\right) v$-almost everywhere. Deducing the $L^{p}$ boundedness of $g_{N}$ by referring to one-dimensional results and Minkowski's integral inequality fails. However, we can show that $g_{N}$ is indeed bounded on $L^{p}$ by using the Plancherel formula (2.8) for the Mellin transform and the appropriate bounds for the $L^{p}$ norms of the one-dimensional imaginary power operators $\mathcal{L}_{n}^{i u}, n=1,2$, stated in the condition (2.11).

Theorem 2.4 Let $1<p<\infty$, then

$$
\|f\|_{p} \lesssim\left\|g_{N}(f)\right\|_{p} \lesssim\|f\|_{p}
$$

The proofs of Theorems 2.2 and 2.4 are given in the next section.

\section{Proofs of Theorems 2.2 and 2.4}

In order to prove Theorem 2.4 we will use the following lemma.

Lemma 3.1 Let $N$ be a positive integer and fix $f \in L^{2}$. Then for a.e. $x=\left(x_{1}, x_{2}\right) \in$ $A_{1} \times A_{2}$, the identity

$$
\mathcal{M}\left(t^{N}{ }_{s}^{N}\left(\frac{d^{N}}{d t^{N}} T_{t}^{1} \frac{d^{N}}{d s^{N}} T_{s}^{2} f\right)(x)\right)\left(u_{1}, u_{2}\right)=\Gamma\left(N-i u_{1}\right) \Gamma\left(N-i u_{2}\right)\left(\mathcal{L}^{i u_{1}, i u_{2}} f\right)(x),
$$

holds $u=\left(u_{1}, u_{2}\right)$-a.e.

Proof The Mellin transform above is that of a function

$$
(t, s) \rightarrow t^{N} s^{N}\left(\frac{d^{N}}{d t^{N}} T_{t}^{1} \frac{d^{N}}{d s^{N}} T_{s}^{2} f\right)(x)
$$


Note that the expression $\mathcal{M}\left(t^{N} s^{N}\left(\frac{d^{N}}{d t^{N}} T_{t}^{1} \frac{d^{N}}{d s^{N}} T_{s}^{2} f\right)(x)\right)$ makes sense, since from Proposition 2.3 we know in particular that the function defined by (3.1) is $x$-a.e. an element of $L^{2}\left((0, \infty)^{2}, \frac{d t}{t} \frac{d s}{s}\right)$. It suffices to verify that the left and the right hand sides agree as elements of the space $L^{2}\left((A, v), L^{2}\left(\mathbb{R}^{2}\right)\right)$. Fix $f \in L^{2}$, with the expansion $\sum_{k \in \mathbb{N}^{2}} a_{k} e_{k}$ and for $\varepsilon>0$ and a.e. $x \in A$, denote

$$
\begin{gathered}
F^{x}=F^{x}(s, t)=t^{N} s^{N}\left(\frac{d^{N}}{d t^{N}} T_{t}^{1} \frac{d^{N}}{d s^{N}} T_{s}^{2} f\right)(x)=F_{N}(f)(t, s), \\
F_{\varepsilon}^{x}=\chi_{\left(\varepsilon, \varepsilon^{-1}\right)}(t) \chi_{\left(\varepsilon, \varepsilon^{-1}\right)}(s) F^{x}(s, t), \quad \mathcal{M}_{\varepsilon}(F)(u)=\mathcal{M}\left(F_{\varepsilon}^{x}\right)(u) .
\end{gathered}
$$

Then since $F_{\varepsilon}^{x} \rightarrow F^{x}$ in $L^{2}\left((0, \infty)^{2}, \frac{d t}{t} \frac{d s}{s}\right)$ as $\varepsilon \rightarrow 0^{+}$, in view of (2.8), we also have

$$
\lim _{\varepsilon \rightarrow 0^{+}} \mathcal{M}\left(F_{\varepsilon}^{x}\right)(u)=\mathcal{M}\left(F^{x}\right)(u),
$$

in $L^{2}\left(\mathbb{R}^{2}, d u\right)$, for a.e. $x \in A$. Hence

$$
\begin{aligned}
& \left\|\mathcal{M}\left(F^{x}\right)(u)-\Gamma\left(N-i u_{1}\right) \Gamma\left(N-i u_{2}\right)\left(\mathcal{L}^{i u_{1}, i u_{2}} f\right)(x)\right\|_{L^{2}\left((A, v), L^{2}\left(\mathbb{R}^{2}\right)\right)}^{2} \\
& =\int_{A} \lim _{R \rightarrow \infty}\left(\lim _{\varepsilon \rightarrow 0} \int_{\mathbb{R}^{2} \cap B_{R}}\left|\mathcal{M}\left(F_{\varepsilon}^{x}\right)(u)-\Gamma\left(N-i u_{1}\right) \Gamma\left(N-i u_{2}\right)\left(\mathcal{L}^{i u_{1}, i u_{2}} f\right)(x)\right|^{2} d u\right) d v \\
& \leq \liminf _{R \rightarrow \infty}\left(\liminf _{\varepsilon \rightarrow 0} \int_{\mathbb{R}^{2} \cap B_{R}} \int_{A}\left|\mathcal{M}_{\varepsilon}(F)(u)-\Gamma\left(N-i u_{1}\right) \Gamma\left(N-i u_{2}\right) \mathcal{L}^{i u_{1}, i u_{2}} f\right|^{2} d v d u\right),
\end{aligned}
$$

where we used Fubini's theorem and Fatou's lemma twice in the inequality above. The symbol $B_{R}$ above denotes the Euclidean ball in $\mathbb{R}^{2}$ with the radius $R$ and the center at the origin. It can be now easily checked that

$$
\mathcal{M}_{\varepsilon}(F)(u)=\sum_{k \in \mathbb{N}^{2}} \Gamma_{\varepsilon, k_{1}}\left(N-i u_{1}\right) \Gamma_{\varepsilon, k_{2}}\left(N-i u_{2}\right)\left(\lambda_{k_{1}}\right)^{i u_{1}}\left(\lambda_{k_{2}}\right)^{i u_{2}} a_{k} e_{k},
$$

where $\Gamma_{\varepsilon, k_{n}}\left(N-i u_{n}\right)=\int_{\varepsilon \lambda_{k_{n}}}^{\varepsilon^{-1} \lambda_{k_{n}}} \tau^{N-i u_{n}-1} e^{-\tau} d \tau, n=1,2$, is the truncated Gamma function. Obviously,

$\Gamma\left(N-i u_{1}\right) \Gamma\left(N-i u_{2}\right) \mathcal{L}^{i u_{1}, i u_{2}} f=\sum_{k \in \mathbb{N}^{2}} \Gamma\left(N-i u_{1}\right) \Gamma\left(N-i u_{2}\right)\left(\lambda_{k_{1}}^{1}\right)^{i u_{1}}\left(\lambda_{k_{2}}^{2}\right)^{i u_{2}} a_{k} e_{k}$ 
Consequently,

$$
\begin{aligned}
& \int_{\mathbb{R}^{2} \cap B_{R}} \int_{A}\left|\mathcal{M}_{\varepsilon}(F)(u)-\Gamma\left(N-i u_{1}\right) \Gamma\left(N-i u_{2}\right) \mathcal{L}^{i u_{1}, i u_{2}} f\right|^{2} d v d u \\
\leq & \sum_{k \in \mathbb{N}^{2}}\left|a_{k}\right|^{2} \int_{\mathbb{R}^{2} \cap B_{R}}\left|\Gamma_{\varepsilon, k_{1}}\left(N-i u_{1}\right) \Gamma_{\varepsilon, k_{2}}\left(N-i u_{2}\right)-\Gamma\left(N-i u_{1}\right) \Gamma\left(N-i u_{2}\right)\right|^{2} d u .
\end{aligned}
$$

The latter quantity goes to zero as $\varepsilon \rightarrow 0^{+}$by the dominated convergence theorem. Coming back to (3.3) we get the desired conclusion.

We now pass to the proof of Theorem 2.4. From Lemma 3.1 and the Plancherel formula (2.8), for $f \in L^{2} \cap L^{p}$ and a.e. $x=\left(x_{1}, x_{2}\right)$, we write

$$
\left(g_{N}(f)(x)\right)^{2}=\left(4 \pi^{2}\right)^{-1} \int_{\mathbb{R}^{2}}\left|\Gamma\left(N-i u_{1}\right) \Gamma\left(N-i u_{2}\right)\left(\mathcal{L}^{i u_{1}, i u_{2}} f\right)(x)\right|^{2} d u
$$

If $p>2$, from Minkowski's integral inequality it follows that

$$
\begin{aligned}
2 \pi\left\|g_{N}(f)\right\|_{p} & \left.=\left(\iint_{A}\left(\int_{\mathbb{R}^{2}}\left|\Gamma\left(N-i u_{1}\right) \Gamma\left(N-i u_{2}\right)\left(\mathcal{L}^{i u_{1}, i u_{2}} f\right)(x)\right|^{2}\right)^{p / 2} d \nu(x)\right)^{2 / p}\right)^{1 / 2} \\
& \lesssim\left(\int_{\mathbb{R}^{2}}\left|\Gamma\left(N-i u_{1}\right) \Gamma\left(N-i u_{2}\right)\right|^{2}\left(\int_{A}\left|\left(\mathcal{L}^{i u_{1}, i u_{2}} f\right)(x)\right|^{p} d \nu(x)\right)^{2 / p} d u\right)^{1 / 2} \\
& \lesssim\left(\int_{\mathbb{R}^{2}}\left|\Gamma\left(N-i u_{1}\right) \Gamma\left(N-i u_{2}\right)\right|^{2}\left\|\mathcal{L}^{i u_{1}, i u_{2}}\right\|_{p \rightarrow p}^{2} d u\right)^{1 / 2}\|f\|_{p} .
\end{aligned}
$$

If $1<p<2$, then using the fact that for nonnegative real numbers, $a, b,(a+b)^{p / 2} \leq$ $a^{p / 2}+b^{p / 2}$ it can be proved (the first inequality below) that

$$
\begin{aligned}
\left\|g_{N}(f)\right\|_{p}^{p} & =(2 \pi)^{-p}\left(\int_{A}\left(\int_{\mathbb{R}^{2}}\left|\Gamma\left(N-i u_{1}\right) \Gamma\left(N-i u_{2}\right)\left(\mathcal{L}^{i u_{1}, i u_{2}} f\right)(x)\right|^{2}\right)^{p / 2} d v(x)\right) \\
& \leq \int_{\mathbb{R}^{2}}\left|\Gamma\left(N-i u_{1}\right) \Gamma\left(N-i u_{2}\right)\right|^{p} \int_{A}\left|\left(\mathcal{L}^{i u_{1}, i u_{2}} f\right)(x)\right|^{p} d v(x) d u \\
& \left.\leq \int_{\mathbb{R}^{2}}\left|\Gamma\left(N-i u_{1}\right) \Gamma\left(N-i u_{2}\right)\right|^{p}\left\|\mathcal{L}^{i u_{1}, i u_{2}}\right\|_{p \rightarrow p}^{p} d u\right)\|f\|_{p}^{p .}
\end{aligned}
$$


Since $|\Gamma(N-i v)| \leq(1+|v|)^{N-1} e^{-\frac{\pi}{2}|v|}, v \in \mathbb{R}$ (see [10, Chapter 1]), using (2.5) and the assumption (2.11) we see that in either case $\left\|g_{N}(f)\right\|_{p} \lesssim\|f\|_{p}$, which is the right hand side inequality in Theorem 2.4. Now the left hand side inequality can be proved in a standard manner, by referring to the right hand side and Lemma 2.3.

Having proved Theorem 2.4 we pass to the proof of Theorem 2.2. We proceed as in [13]. We need the following lemma (appearing in a similar, one-dimensional form in [13]).

Lemma 3.2 Let $f \in L^{2}$. For $t, s>0$ we have

$$
F_{N+1}(m(\mathcal{L}) f)(t, s)=\frac{1}{4 \pi^{2}} \int_{\mathbb{R}^{2}} \mathcal{M}\left(m_{N, t / 2, s / 2}\right)\left(u_{1}, u_{2}\right) t s \frac{d}{d t} T_{t / 2}^{1} \frac{d}{d s} T_{s / 2}^{2}\left(\mathcal{L}^{i u_{1}, i u_{2}} f\right) d u
$$

where $F_{N+1}(t, s)$ is defined by (3.2) and the right hand side is understood as a Bochner integral taking values in $L^{2}$.

Proof Fix $t, s>0$ and let $f, g \in L^{2}, f \sim \sum_{k} a_{k} e_{k}, g \sim \sum_{k} b_{k} e_{k}$. Then

$$
\begin{aligned}
& 4 \pi^{2}\left\langle F_{N+1}(m(\mathcal{L}) f)(t, s), g\right\rangle \\
& =\sum_{k \in \mathbb{N}^{2}} m_{N, t / 2, s / 2}\left(\lambda_{k_{1}}, \lambda_{k_{2}}\right)\left(\lambda_{k_{1}} t\right)\left(\lambda_{k_{2}} s\right) e^{-t \lambda_{k_{1}} / 2} e^{-s \lambda_{k_{2}} / 2} a_{k} b_{k} \\
& =\sum_{k \in \mathbb{N}^{2}} \int_{\mathbb{R}^{2}} \mathcal{M}\left(m_{N, t / 2, s / 2}\right)\left(u_{1}, u_{2}\right) \lambda_{k_{1}}^{i u_{1}} \lambda_{k_{2}}^{i u_{2}} d u\left(\lambda_{k_{1}} t\right)\left(\lambda_{k_{2}} s\right) e^{-t \lambda_{k_{1}} / 2} e^{-s \lambda_{k_{2}} / 2} a_{k} b_{k} \\
& =\int_{\mathbb{R}^{2}} \mathcal{M}\left(m_{N, t / 2, s / 2}\right)\left(u_{1}, u_{2}\right)\left\langle t s \frac{d}{d t} T_{t / 2}^{1} \frac{d}{d s} T_{s / 2}^{2}\left(\mathcal{L}^{i u_{1}, i u_{2}} f\right), g\right\rangle d u,
\end{aligned}
$$

where in the second equality above we used the inversion formula (2.7), and in the third we used the dominated convergence theorem and the identity (2.2).

Using Theorem 2.4 (the first and third inequalities below), the above lemma (the second equality below) and Minkowski's integral inequality twice (the second inequality below), we finally write

$$
\begin{aligned}
& \|m(\mathcal{L}) f\|_{p} \lesssim 4 \pi^{2}\left\|g_{N+1}(m(\mathcal{L}) f)\right\|_{p} \\
& =4 \pi^{2}\left\|\left(\int_{0}^{\infty} \int_{0}^{\infty}\left|F_{N+1}(m(\mathcal{L}) f)(t, s)\right|^{2} \frac{d t}{t} \frac{d s}{s}\right)^{1 / 2}\right\|_{p} \\
& =\left\|\left(\int_{0}^{\infty} \int_{0}^{\infty}\left|\int_{\mathbb{R}^{2}} \mathcal{M}\left(m_{N, t / 2, s / 2}\right)\left(u_{1}, u_{2}\right) t s \frac{d}{d t} T_{t / 2}^{x} \frac{d}{d s} T_{s / 2}^{y}\left(\mathcal{L}^{i u_{1}, i u_{2}} f\right) d u\right|^{2} \frac{d t}{t} \frac{d s}{s}\right)^{1 / 2}\right\|_{p}
\end{aligned}
$$




$$
\begin{aligned}
& \lesssim \int_{\mathbb{R}^{2}} \sup _{t, s>0}\left|\mathcal{M}\left(m_{N, t, s}\right)\left(u_{1}, u_{2}\right)\right|\left\|g_{1}\left(\mathcal{L}^{i u_{1}, i u_{2}} f\right)\right\|_{p} d u \\
& \lesssim \int_{\mathbb{R}^{2}} \sup _{t, s>0}\left|\mathcal{M}\left(m_{N, t, s}\right)\left(u_{1}, u_{2}\right)\right|\left\|\mathcal{L}^{i u_{1}, i u_{2}}\right\|_{p \rightarrow p} d u\|f\|_{p} .
\end{aligned}
$$

The proof of Theorem 2.2 is thus finished.

\section{The polynomial growth case and applications}

We proceed as in the proof of [13, Theorem 4]. Throughout this section we assume that the norms of the one-dimensional imaginary power operators have at most polynomial growth, more precisely, there exists a vector of positive real numbers $\sigma=$ $\left(\sigma_{1}, \ldots, \sigma_{d}\right)$, such that

$$
\left\|\mathcal{L}_{n}^{i v}\right\|_{p \rightarrow p} \lesssim(1+|v|)^{\sigma_{n}|1 / p-1 / 2|}, \quad v \in \mathbb{R}, \quad n=1, \ldots, d
$$

which in view of (2.6) gives

$$
\left\|\mathcal{L}^{i u_{1}, \ldots, i u_{d}}\right\|_{p \rightarrow p} \lesssim \prod_{n=1}^{d}\left(1+\left|u_{n}\right|\right)^{\sigma_{n}|1 / p-1 / 2|}
$$

We say that $m$ satisfies Marcinkiewicz's condition of order $\rho$ if $m$ is bounded and for all multi-indices $\gamma=\left(\gamma_{1}, \ldots, \gamma_{d}\right) \leq \rho$

$$
\sup _{R_{1}, \ldots, R_{d}>0} \int_{R_{1}<\lambda_{1}<2 R_{1}} \cdots \int_{R_{d}<\lambda_{d}<2 R_{d}}\left|\lambda^{\gamma} D^{\gamma} m(\lambda)\right|^{2} \frac{d \lambda}{\lambda}<\infty .
$$

Theorem 4.1 Assume that m satisfies the Marcinkiewicz condition (4.2) of some order $\rho>|1 / p-1 / 2| \sigma+\mathbf{1}$, with some $1<p<\infty$. Then $m(\mathcal{L})$ extends to a bounded operator on $L^{p}$. In particular, if $\rho>\sigma / 2+1$, then $m(\mathcal{L})$ extends to a bounded operator on all $L^{p}$ spaces, $1<p<\infty$.

Proof As previously we will only prove the case $d=2$. Proceeding as in [13] we show that $m$ satisfies (2.10). Let $N$ be an integer greater then $\rho$. Let $\psi$ be a nonnegative, $C^{\infty}$ function supported in $[1 / 2,2]$ such that

$$
\sum_{j=-\infty}^{\infty} \psi_{j}(\xi)=1, \quad \xi>0
$$

where $\psi_{j}(\xi)=\psi\left(2^{j} \xi\right)$. Then, obviously

$$
\sum_{j, l} \psi_{j, l}(\xi, \eta)=1, \quad(\lambda, \eta) \in \mathbb{R}_{+}^{2},
$$


where $\psi_{j, l}(\xi, \eta)=\psi_{j}(\lambda) \psi_{l}(\eta)$. Set

$$
c_{N, r, u}=\frac{(-1)^{r}}{\left(N-i u_{1}\right) \cdots\left(N-i u_{1}+r-1\right)}, \quad r \in \mathbb{N} .
$$

Integrating by parts $\rho_{1}$ times in the first variable and $\rho_{2}$ times in the second variable we see that $\mathcal{M}\left(m_{N, t, s}\right)\left(u_{1}, u_{2}\right)$ equals $c_{N, \rho_{1}, u_{1}} c_{N, \rho_{2}, u_{2}}$ times

$$
t^{i u_{1}} s^{i u_{2}} \sum_{j, l} \int_{0}^{\infty} \int_{0}^{\infty} \xi^{N+\rho_{1}-i u_{1}} \eta^{N+\rho_{2}-i u_{2}} D^{\rho_{1}, \rho_{2}}\left(e^{-\xi-\eta} m(\xi / t, \eta / s) \psi_{j, l}(\xi, \eta)\right) \frac{d \xi}{\xi} \frac{d \eta}{\eta} .
$$

Leibniz's rule allows us to express the derivative $D^{\rho_{1}, \rho_{2}}$ as a weighted sum of derivatives of the form

$$
\begin{aligned}
E_{\gamma, \delta, t, s}^{j, l}(\xi, \eta)= & e^{-\xi-\eta} t^{-\gamma_{1}} s^{-\gamma_{2}}\left(D^{\gamma} m\right)(\xi / t, \eta / s) 2^{j \delta_{1}} 2^{l \delta_{2}} \\
& \times\left(\frac{d^{\delta_{1}}}{d \xi^{\delta_{1}}} \psi\right)\left(2^{j} \xi\right)\left(\frac{d^{\delta_{2}}}{d \eta^{\delta_{2}}} \psi\right)\left(2^{l} \eta\right),
\end{aligned}
$$

where $\gamma=\left(\gamma_{1}, \gamma_{2}\right)$ and $\delta=\left(\delta_{1}, \delta_{2}\right)$ are multi-indices such that $\gamma+\delta \leq \rho$. Proceeding further as in [13], we define

$$
I_{j, l, N, \rho}(t, s) \equiv \int_{0}^{\infty} \int_{0}^{\infty} \xi^{N+\rho_{1}-i u_{1}} \eta^{N+\rho_{2}-i u_{2}} E_{\gamma, \delta, t, s}^{j, l}(\xi, \eta) \frac{d \xi}{\xi} \frac{d \eta}{\eta} .
$$

An argument analogous to the one used in [13] shows that it is sufficient to verify that

$$
\left|I_{j, l, N, \rho}(t, s)\right| \lesssim p_{j}^{1} p_{l}^{2}
$$

with $(n=1,2)$

$$
p_{j}^{n}= \begin{cases}2^{-j \rho_{n}}, & \text { if } j>0 \\ 2^{-j\left(N+\rho_{n}\right)} \exp \left(-2^{-k-1}\right), & \text { if } j \leq 0\end{cases}
$$

Now the change of variable $\left(2^{j} \xi, 2^{l} \eta\right) \rightarrow(\xi, \eta)$ allows us to repeat the final steps of the proof of $[13,(11)]$, leading to the bound

$$
\sup _{t, s>0}\left|\mathcal{M}\left(m_{N, t, s}\right)\left(u_{1}, u_{2}\right)\right| \lesssim\left(1+\left|u_{1}\right|\right)^{-\rho_{1}}\left(1+\left|u_{2}\right|\right)^{-\rho_{2}}
$$

Combining the latter with the assumptions (2.10) and (4.1) we get the desired conclusion. 
Theorem 4.1 seems to be the most applicable. In many cases we do know the bounds for the norms of the imaginary power operators. For instance, if the operator $\mathcal{L}_{n}$ satisfies the assumptions of [17, Theorem 2] with $d=D=l_{n}$, then

$$
\left\|\mathcal{L}_{n}^{i v}\right\|_{p} \lesssim(1+|v|)^{l_{n}|1 / p-1 / 2|}, \quad v \in \mathbb{R} .
$$

This is the case with the operators corresponding to the Hermite expansions and the Laguerre expansions of Hermite type, which satisfy (4.3) with $l_{n}$ equal to the Euclidean dimension of the space on which they act. It is noteworthy that even for the Hermite expansions the theorem we can obtain seems to be slightly different in nature than that of Thangavelu [22,23], since in our case it has differential, non difference form.

As another application we shall derive a multiplier theorem for the Jacobi expansions by showing that the norms of the one-dimensional imaginary power operators connected with them have polynomial growth and a reference to Theorem 4.1. Denote $\log _{+}(z)=\max (1, \log (z)), z \geq 0$ (with the convention $\log _{+}(0)=1$ ). For parameters $\alpha, \beta>-1$, consider the Jacobi operator

$$
\mathcal{J}^{\alpha, \beta}=-\frac{d}{d \theta^{2}} \frac{\alpha-\beta+(\alpha+\beta+1) \cos \theta}{\sin \theta} \frac{d}{d \theta}+\left(\frac{\alpha+\beta+1}{2}\right)^{2},
$$

on the interval $(0, \pi)$ equipped with the measure

$$
d \mu_{\alpha, \beta}(\theta)=\left(\sin \frac{\theta}{2}\right)^{2 \alpha+1}\left(\cos \frac{\theta}{2}\right)^{2 \beta+1} d \theta
$$

In this case not much is known about the heat semigroup generated by $\mathcal{J}^{\alpha, \beta}$. In particular we do not know if there are Gaussian bounds for it. Let $\mathcal{P}_{r}^{\alpha, \beta}(\theta), \theta \in(0, \pi)$, be the system of Jacobi trigonometric polynomials, as defined in [16]. It is well known that $\left\{\mathcal{P}_{r}^{\alpha, \beta}\right\}_{r \in \mathbb{N}}$ forms an orthonormal basis in $\left((0, \pi), d \mu_{\alpha, \beta}\right)$. Since a self adjoint extension of the operator $\mathcal{J}^{\alpha, \beta}$ is given by

$$
\tilde{\mathcal{J}}^{\alpha, \beta} f=\sum_{r=0}^{\infty}\left(r+\frac{\alpha+\beta+1}{2}\right)^{2}\left\langle f, \mathcal{P}_{r}^{\alpha, \beta}\right\rangle_{\mu_{\alpha, \beta}} \mathcal{P}_{r}^{\alpha, \beta},
$$

we are lead to define the Jacobi imaginary power operators by

$$
I_{\gamma}^{\alpha, \beta} f=\sum_{r=0}^{\infty}\left|r+\frac{\alpha+\beta+1}{2}\right|^{-2 \gamma i}\left\langle f, \mathcal{P}_{r}^{\alpha, \beta}\right\rangle_{\mu_{\alpha, \beta}} \mathcal{P}_{r}^{\alpha, \beta}, \quad \gamma \in \mathbb{R}, \quad \gamma \neq 0 .
$$

Note that for $\alpha=\beta=(d-3) / 2, d \geq 2$, using the connection between the Jacobi (Gegenbauer) polynomials and the spherical Laplacian on the $d$-dimensional unit sphere, we can obtain polynomial bounds for the norms of the Jacobi imaginary power operators, see the remarks at the end of [17]. In the general case, we have the following. 
Proposition 4.2 Assume that $\alpha, \beta \geq-1 / 2, \alpha+\beta>-1, \gamma \in \mathbb{R}, \gamma \neq 0$. Then

$$
\left\|I_{\gamma}^{\alpha, \beta}\right\|_{p \rightarrow p} \leq C_{\alpha, \beta} \log _{+}(|\gamma|)^{|2 / p-1|}(1+|\gamma|)^{(4 \alpha+4 \beta+11)|1 / p-1 / 2|}
$$

Proof In the inequality above the symbol $\|\cdot\|_{p \rightarrow p}$ represents the operator norm on the space $L^{p}\left((0, \pi), \mu_{\alpha, \beta}\right)$. To prove the proposition we will use the subtle estimates obtained by Nowak and Sjögren [16] and some complex variable techniques as in [9]. By referring to the Calderón-Zygmund theory and the Marcinkiewicz interpolation theorem we see that it suffices to show that the Calderón-Zygmund constant $D_{\gamma}$ in the smoothness condition for the kernel $K_{\gamma}^{\alpha, \beta}(\theta, \phi)$, defined by [16, (12) p. 8], is less than a constant times $\log _{+}(|\gamma|)(1+|\gamma|)^{2 \alpha+2 \beta+\frac{11}{2}}$. Since the function $\gamma \rightarrow \frac{1}{\Gamma(2 i \gamma)}$ is continuous (see for instance [10, Chapter 1]), looking closely at the prove of the smoothness bound from [16, p. 20] we see that it suffices to focus on $|\gamma|>1$. For symmetry reasons we may consider only the derivatives with respect to the variable $\theta$. By [16, Proposition 4.1],

$$
\begin{aligned}
\Gamma(2 i \gamma) \frac{\partial}{\partial \theta} I_{\gamma}^{\alpha, \beta}= & \int_{0}^{\infty} t^{2 i \gamma-1} \sinh \frac{t}{2} \iint d \Pi_{\alpha}(u) d \Pi_{\beta}(v) \\
& \times \frac{\partial_{\theta} q}{\left(\cosh \frac{t}{2}-1+q\right)^{\alpha+\beta+3}} d t \equiv J_{\gamma}
\end{aligned}
$$

with

$$
d \Pi_{\alpha}(u)=\frac{\Gamma(\alpha+1)}{\sqrt{\pi} \Gamma(\alpha+1 / 2)}\left(1-u^{2}\right)^{\alpha-1 / 2} d u,
$$

and $q$ being defined in [16, pp. 16-17] (note that $0 \leq q \leq 2$ ). Assume $\gamma>1$ and set $\frac{\pi}{4}<\phi<\frac{\pi}{2}$. We consider the function

$$
h(z)=z^{2 i \gamma-1} \sinh \frac{z}{2} \iint d \Pi_{\alpha}(u) d \Pi_{\beta}(v) \frac{\partial_{\theta} q}{\left(\cosh \frac{z}{2}-1+q\right)^{\alpha+\beta+3}}
$$

which is holomorphic in the right half plane. Then we change the contour of integration in the integral formula for $J_{\gamma}$ to $\left(e^{i \phi} 0, e^{i \phi} \infty\right)$ (the other integrals can be easily seen to vanish), where for an angle $\phi$, by $\left(0 e^{i \phi}, \infty e^{i \phi}\right)$ we mean the ray $\left\{t e^{i \phi}: t \geq 0\right\}$. Of course $h$ is not holomorphic at zero, but this difficulty can be easily overcome by a limiting process. Using the parametrization $\gamma(t)=t e^{i \phi}, \gamma^{\prime}(t)=e^{i \phi}$, we get

$$
\begin{aligned}
\left|J_{\gamma}\right| & \leq e^{-2 \gamma \phi} \int_{0}^{\infty} t^{-1}\left|\sinh \frac{\gamma(t)}{2}\right| \iint d \Pi_{\alpha}(u) d \Pi_{\beta}(v) \frac{\partial_{\theta} q}{\left|\cosh \frac{\gamma(t)}{2}-1+q\right|^{\alpha+\beta+3}} d t \\
& =e^{-2 \gamma \phi}\left(\int_{0}^{1} \cdots+\int_{1}^{\frac{2}{\cos \phi}} \cdots+\int_{\frac{2}{\cos \phi}}^{\infty}\right) \equiv e^{-2 \gamma \phi}\left(J_{1}+J_{2}+J_{3}\right) .
\end{aligned}
$$


In the reasoning below the symbol $\lesssim$ indicates that the constant in the inequality is independent of both $\gamma$ and $\phi$. We start with estimating $J_{1}$. Let

$$
A(t)=\cosh \frac{\gamma(t)}{2}=\cosh (t \cos \phi / 2) \cos (t \sin \phi / 2)+i \sinh (t \cos \phi / 2) \sin (t \sin \phi / 2),
$$

and let $B(t)=A(t)-1+q$. Clearly, since $\sin \phi / 2>1 / 4$ and $\sinh x>\frac{1}{2} x, \sin x>$ $\frac{1}{2} x$, for $0<x \leq 1$, we see that $\operatorname{Im}(B(t)) \geq(\cos \phi) t^{2} / 4$, for $0<t \leq 1$. Consequently, if $t^{2} / 16>\frac{1}{2} q$, then $\cos \phi\left(t^{2}+q\right) \lesssim|B(t)|$. On the other hand, if $t^{2} / 16 \leq \frac{1}{2} q$, then since $1-x^{2} \leq \cos x$ and $(\sin \phi)^{2} \geq 1 / 2$, we see that $\operatorname{Re}(B(t)) \geq-t^{2} / 16+q \geq \frac{1}{2} q$. Hence, in either case $\cos \phi\left(t^{2}+q\right) \lesssim|B(t)|$. From the latter inequality, together with the bound $|\gamma(t) \sinh (\gamma(t) / 2)| \lesssim 1,0<t<1$, we get

$$
J_{1} \lesssim(\cos \phi)^{-\alpha-\beta-3} \int_{0}^{1} \iint d \Pi_{\alpha}(u) d \Pi_{\beta}(v) \frac{\partial_{\theta} q}{\left(t^{2}+q\right)^{\alpha+\beta+3}} d t
$$

Now we pass to the estimation of $J_{2}$. Let $\delta^{2}=\cosh (\cos \phi / 2)$. From (4.4), the inequality $|-1+q| \leq 1$ and the fact that $1+(\cos \phi / 2)^{2} / 2 \lesssim \delta^{2} \approx 1$, we see that if $\cos (t \sin \phi / 2) \geq \frac{1}{\delta}$, then $|\operatorname{Re}(B(t))| \geq C(\cos (\phi))^{2}$; whereas if $\cos (t \sin \phi / 2)<$ $\frac{1}{\delta}$, then $\mid \operatorname{Im}\left(B(t) \mid \geq C(\cos (\phi))^{2}\right.$. In either case, $|B(t)| \geq C(\cos \phi)^{2}$, so that since $|\sinh (\gamma(t) / 2)| \lesssim 1$, for $t \in\left(1, \frac{2}{\cos \phi}\right)$, and $q, \partial_{\theta} q$ are bounded we obtain

$$
J_{2} \lesssim \log _{+}(\cos \phi)(\cos \phi)^{-2 \alpha-2 \beta-6} \iint \frac{d \Pi_{\alpha}(u) d \Pi_{\beta}(v)}{q^{\alpha+\beta+2}} .
$$

We now pass to $J_{3}$. Since, $|A(t)|^{2}=(\cosh (t \cos \phi / 2))^{2}-(\sin (t \sin \phi / 2))^{2}$ we see that for $t \geq \frac{2}{\cos \phi},|B(t)| \geq C e^{t \cos \phi / 2}$. Similarly, for such $t$ we have

$$
|\sinh (\gamma(t) / 2)|^{2}=(\sinh (t \cos \phi / 2))^{2}+(\sin (t \sin \phi / 2))^{2} \lesssim e^{t \cos \phi} .
$$

Combining the latter two inequalities together with the boundedness of $q, \partial_{\theta} q$, and the change of variable $t \cos \phi \rightarrow t$, we obtain

$$
J_{3} \lesssim \int_{1}^{\infty} e^{-t / 2(\alpha+\beta+2)} \iint \frac{d \Pi_{\alpha}(u) d \Pi_{\beta}(v)}{q^{\alpha+\beta+2}} d t
$$

From the estimates (4.5), (4.6) and (4.7) proceeding as in [16] (in the case of the bound (4.5) we need to refer to [16, Lemma 4.5]), for $\frac{\pi}{4}<\phi<\frac{\pi}{2}$, we derive the bound independent of $\gamma>1$,

$$
\left|J_{\gamma}\right| \lesssim \log _{+}(\cos \phi)(\cos \phi)^{-2 \alpha-2 \beta-6} \iint \frac{d \Pi_{\alpha}(u) d \Pi_{\beta}(v)}{q^{\alpha+\beta+2}} .
$$


Now, from [16, Lemma 4.3] it follows that the Calderón-Zygmund constant $D_{\gamma}$ in the smoothness condition for the kernel $K_{\gamma}^{\alpha, \beta}$ satisfies

$$
D_{\gamma} \lesssim \frac{e^{-2 \gamma \phi}}{\Gamma(2 i \gamma)} \log _{+}(\cos \phi)(\cos \phi)^{-2 \alpha-2 \beta-6}
$$

Since $(1+\gamma)^{1 / 2} e^{-\pi \gamma} \lesssim|\Gamma(2 i \gamma)|$ (see for instance [10, Chapter 1]), taking $\phi=$ $\arctan (\gamma)$ and using (4.8) we finally arrive at

$$
D_{\gamma} \lesssim \log _{+}(\gamma)(1+\gamma)^{2 \alpha+2 \beta+\frac{11}{2}} \exp \left(2\left(\frac{\pi}{2}-\arctan (\gamma)\right) \gamma\right)
$$

which easily produces the desire bound in the case $\gamma>0$. The reasoning in the case $\gamma<-1$ is analogous, we need only to replace the ray $\left(0 e^{i \phi}, \infty e^{i \phi}\right)$ by $\left(0 e^{-i \phi}, \infty e^{-i \phi}\right)$.

Let

$$
\mathcal{P}_{k}^{\alpha, \beta}(\theta)=\mathcal{P}_{k_{1}}^{\alpha_{1}, \beta_{1}}\left(\theta_{1}\right) \ldots \mathcal{P}_{k_{d}}^{\alpha_{d}, \beta_{d}}\left(\theta_{d}\right), \quad \theta=\left(\theta_{1}, \ldots, \theta_{d}\right) \in(0, \pi)^{d}
$$

with $\alpha=\left(\alpha_{1}, \ldots, \alpha_{d}\right), \beta=\left(\beta_{1}, \ldots, \beta_{d}\right), \alpha+\beta>\mathbf{- 1}$, be the system of $d$-dimensional Jacobi trigonometric polynomials. Analogously as in the one-dimensional case, $\left\{\mathcal{P}_{k}^{\alpha, \beta}\right\}_{k \in \mathbb{N}^{d}}$ forms an orthonormal basis in $\left((0, \pi)^{d}, d \mu_{\alpha, \beta}\right)$, with

$$
d \mu_{\alpha, \beta}(\theta)=d \mu_{\alpha_{1}, \beta_{1}}\left(\theta_{1}\right) \times \cdots \times d \mu_{\alpha_{d}, \beta_{d}}\left(\theta_{d}\right) .
$$

For a bounded function $m$ on $(0, \infty)^{d}$ define the multiplier operator $T_{m}^{\alpha, \beta}$ with respect to the system $\mathcal{P}_{k}^{\alpha, \beta}$ according to (2.1). Using Proposition 4.2 together with Theorem 4.1 we immediately get the following.

Corollary 4.3 Let $\alpha+\beta>-1, \alpha, \beta \geq-\mathbf{1} / 2$. Assume that the function $m$ satisfies the Marcinkiewicz condition (4.2) of some order $\rho>|1 / p-1 / 2|(4 \alpha+4 \beta+\mathbf{1 1})+\mathbf{1}$, with some $1<p<\infty$. Then the multiplier operator $T_{m}^{\alpha, \beta}$ extends to a bounded operator on $L^{p}\left((0, \pi)^{d}, d \mu_{\alpha, \beta}\right)$. In particular, if $\rho>2 \alpha+2 \beta+\frac{\mathbf{1 3}}{\mathbf{2}}$, then $T_{m}^{\alpha, \beta}$ extends to a bounded operator on all $L^{p}\left((0, \pi)^{d}, d \mu_{\alpha, \beta}\right)$ spaces, $1<p<\infty$.

\section{A multivariate multiplier theorem for the Hankel transform}

In this section we demonstrate that the techniques used in the paper may be also applied in the context of the (modified) Hankel transform multipliers (which may be thought of as an example of a continuous orthogonal expansions). Throughout the rest of the paper the symbol $L^{p}, 1<p<\infty$, will denote the space $L^{p}\left((0, \infty)^{d}, d v_{\alpha}(x)\right)$, with the norm $\|\cdot\|_{p}$ and $d v_{\alpha}(x)=d v_{\alpha_{1}}\left(x_{1}\right) \cdots d v_{\alpha_{d}}\left(x_{d}\right)$, where $d v_{\alpha_{n}}\left(x_{n}\right)=x_{n}^{2 \alpha_{n}} d x_{n}, n=$ 
$1, \ldots, d$. For $\alpha \geq \mathbf{0}$ define the (modified) Hankel transform by

$$
\mathcal{H}_{\alpha}(f)(\lambda)=\int_{(0, \infty)^{d}} E_{\lambda}^{\alpha}(x) f(x) d v_{\alpha}(x), \quad f \in L^{1},
$$

where

$$
E_{\lambda}^{\alpha}(x)=\prod_{n=1}^{d}\left(x_{n} \lambda_{n}\right)^{-\alpha_{n}+1 / 2} J_{\alpha_{n}-1 / 2}\left(x_{n} \lambda_{n}\right)=\prod_{n=1}^{d} E_{\lambda_{n}}^{\alpha_{n}}\left(x_{n}\right) .
$$

Here $J_{v}$ is the Bessel function of the first kind and order $v$, see [10, Chapter 5]. The system $\left\{E_{\lambda}^{\alpha}\right\}_{\lambda \in(0, \infty)^{d}}$ may be thought of as a continuous tensor product orthogonal system in $L^{2}$ that consist of the eigenvectors of the Bessel operator

$$
\Delta_{\alpha}=-\Delta-\sum_{n=1}^{d} \frac{2 \alpha_{n}}{x_{n}} \frac{\partial}{\partial x_{n}}
$$

explicitly, $\Delta_{\alpha}\left(E_{\lambda}^{\alpha}\right)=|\lambda|^{2} E_{\lambda}^{\alpha}$. Also, the functions $E_{\lambda_{n}}^{\alpha_{n}}, n=1, \ldots, d$ are eigenfunctions of the one-dimensional Bessel operators $L_{n}=-\frac{\partial^{2}}{\partial x_{n}{ }^{2}}+\frac{2 \alpha_{n}}{x_{n}} \frac{\partial}{\partial x_{n}}$, explicitly $L_{n}\left(E_{\lambda_{n}}^{\alpha_{n}}\right)=\lambda_{n}^{2} E_{\lambda_{n}}^{\alpha_{n}}$. It can be shown that $\mathcal{H}_{\alpha}$ extends to an isometry of $L^{2}$ that satisfies $\mathcal{H}_{\alpha}^{-1}=\mathcal{H}_{\alpha}$. Moreover, for appropriate $f \in L^{2}$, we have $\Delta_{\alpha}(f)=\mathcal{H}_{\alpha}\left(|x|^{2} \mathcal{H}_{\alpha}\right)(f)$. The latter equation may be used as a definition of a self adjoint extension of the operator $\Delta_{\alpha}$ (which we denote by the same symbol) on the domain

$$
\operatorname{Dom}\left(\Delta_{\alpha}\right)=\left\{f \in L^{2}:|x|^{2} \mathcal{H}_{\alpha}(f) \in L^{2}\right\} .
$$

Let $m:(0, \infty)^{d} \rightarrow \mathbb{C}$ be a bounded measurable function. Define the multiplier operator $T_{m}$ by

$$
T_{m}(f)=\mathcal{H}_{\alpha}\left(m \mathcal{H}_{\alpha} f\right) .
$$

Clearly, $T_{m}$ is bounded on $L^{2}$. Also note that if $m(x)=\widetilde{m}\left(|x|^{2}\right)$, for some bounded, measurable function $\widetilde{m}$ on $(0, \infty)$, then the Hankel multiplier operator defined by $(5.1)$ coincides with the spectral multiplier operator $\widetilde{m}\left(\Delta_{\alpha}\right)$. Let

$$
L_{n}^{i u_{n}} f=\mathcal{H}_{\alpha}\left(\left|x_{n}\right|^{2 i u_{n}} \mathcal{H}_{\alpha} f\right), \quad u_{n} \in \mathbb{R}, \quad n=1, \ldots, d, \quad f \in L^{2} .
$$

Note that the operators $L_{n}^{i u_{n}}$ commute and $L_{n}^{i u_{n}} f=\mathcal{H}_{\alpha_{n}}\left(\left|x_{n}\right|^{2 i u_{n}} \mathcal{H}_{\alpha_{n}} f\right)$, where $\mathcal{H}_{\alpha_{n}}$ is the one dimensional Hankel transform acting on the $n$th variable. Define

$$
L^{i u_{1}, \ldots, i u_{n}} f=\mathcal{H}_{\alpha}\left(\prod_{n=1}^{d}\left|x_{n}\right|^{2 i u_{n}} \mathcal{H}_{\alpha} f\right), \quad f \in L^{2}
$$


Then, since $L^{i u_{1}, \ldots, i u_{n}} f=L_{1}^{i u_{1}} \cdots L_{d}^{i u_{d}} f$, for $f \in L^{p} \cap L^{2}$ an argumentation similar as in the discrete case shows that

$$
\left\|L^{i u_{1}, \ldots, i u_{n}}\right\|_{p \rightarrow p} \leq \prod_{n=1}^{d}\left\|L_{n}^{i u_{n}}\right\|_{p \rightarrow p}
$$

where the symbol $\|\cdot\|_{p \rightarrow p}$ on the right hand side of the above inequality denotes the operator norms on the spaces $L^{2}\left((0, \infty), v_{\alpha_{n}}\right), n=1, \ldots, d$.

Let

$$
S_{\tau}^{n} f=\mathcal{H}_{\alpha}\left(e^{-\tau x_{n}^{2}} \mathcal{H}_{\alpha} f\right), \quad f \in L^{2}\left(A_{n}, v_{n}\right), \quad n=1,2, \ldots, d, \quad \tau>0,
$$

be the one-dimensional heat semigroups corresponding to the operators $L_{n}$ acting on $L^{2}\left(A_{n}, v_{n}\right)$. It is well known that the semigroups have the form

$$
S_{\tau}^{n} f\left(x_{n}\right)=\int_{0}^{\infty} S_{\tau}^{n}\left(x_{n}, y_{n}\right) f\left(y_{n}\right) d v_{n}\left(y_{n}\right), \quad n=1, \ldots, d,
$$

with

$$
S_{\tau}^{n}\left(x_{n}, y_{n}\right)=(2 \tau)^{-1} \exp \left(-\frac{x_{n}^{2}+y_{n}^{2}}{4 \tau}\right) I_{\alpha_{n}-1 / 2}\left(\frac{x_{n} y_{n}}{2 \tau}\right)\left(x_{n} y_{n}\right)^{-\left(\alpha_{n}-1 / 2\right)},
$$

where $I_{v}$ is the (positive) Bessel function of the second kind and order $v$, see [10, Chapter 5]. Using the asymptotics for $I_{\nu}$, together with the integral representation for $S_{\tau}^{n}$ (for the second inequality below), we can show that

$$
\begin{aligned}
& S_{\tau}^{n}\left(x_{n}, y_{n}\right) \lesssim \tau^{-\alpha_{n}-1 / 2} \exp \left(-\frac{\left(x_{n}-y_{n}\right)^{2}}{4 \tau}\right), \quad \tau, x_{n}, y_{n}>0 . \\
& \left\|S_{\tau}^{n}\right\|_{L^{2}\left(A_{n}, v_{n}\right) \rightarrow L^{\infty}}^{2} \lesssim \tau^{-\alpha_{n}-1 / 2}, \quad \tau>0 .
\end{aligned}
$$

Now from [19, Theorem 3] it follows that the inequalities (5.2) and (5.3) together imply the assumption (10) of [17, Theorem 2]. Since

$$
v_{n}\left(\left(x_{n}-R, x_{n}+R\right)\right) \approx R\left(x_{n}+R\right)^{2 \alpha_{n}}, \quad x_{n}, R>0,
$$

we see that $v_{n}\left(\left(x_{n}-b^{-1} R, x_{n}+b^{-1} R\right)\right) \lesssim b^{-2 \alpha_{n}-1} v_{n}\left(\left(x_{n}-R, x_{n}+R\right)\right)$, for $0<b<$ 1. Next, looking in detail at the proof of [17, Theorem 2] we see that its conclusion (with $d=D=\alpha_{n}+1 / 2$ ) holds also in our case, hence

$$
\left\|L_{n}^{i u_{n}}\right\|_{p \rightarrow p} \lesssim\left(1+\left|u_{n}\right|\right)^{\left(2 \alpha_{n}+1\right)|1 / p-1 / 2|}, \quad n=1, \ldots, d .
$$

Consequently, the operator $L^{i u_{1}, \ldots, i u_{n}}$ extends to a bounded operator on $L^{p}$ and

$$
\left\|L^{i u_{1}, \ldots, i u_{n}}\right\|_{p \rightarrow p} \lesssim \prod_{n=1}^{d}\left(1+\left|u_{n}\right|\right)^{\left(2 \alpha_{n}+1\right)|1 / p-1 / 2|}
$$


From now on we proceed as in the discrete tensor product orthogonal case. Define $m_{N, T}$ as in (2.9). The theorems we obtain are the following.

Theorem 5.1 Assume that for some $1<p<\infty$ and some positive integer $N$ the following condition holds

$$
\int_{\mathbb{R}^{d}} \sup _{T \in(0, \infty)^{d}}\left|\mathcal{M}\left(m_{N, T}\right)\left(u_{1}, \ldots, u_{d}\right)\right| \prod_{n=1}^{d}\left(1+\left|u_{n}\right|\right)^{\left(2 \alpha_{n}+1\right)|1 / p-1 / 2|} d u<\infty .
$$

Then the operator $T_{m}$ defined on $L^{2}$ by (5.1) extends to a bounded operator on $L^{p}$.

Theorem 5.2 Assume $1<p<\infty$ and that $m$ satisfies the Marcinkiewicz condition (4.2) of some order $\rho>|1 / p-1 / 2|(2 \alpha+1)+1$. Then the operator $T_{m}$ defined initially on $L^{2}$ by (5.1) extends to a bounded operator on $L^{p}$. In particular, if $\rho>\alpha+\frac{\mathbf{3}}{\mathbf{2}}$, then $T_{m}$ extends to a bounded operator on all $L^{p}$ spaces, $1<p<\infty$.

Note that from (5.4), the analogue of the condition (2.11) from Theorem 2.2 is satisfied in the case of the Hankel transform. Theorem 5.2 can be deduced from Theorem 5.1 exactly as in the discrete orthogonal case.

Outline of the proof of Theorem 5.1. We focus on $d=2$. Then using the onedimensional heat semigroups for the Hankel transform $S_{\tau}^{n} f=\mathcal{H}_{\alpha}\left(e^{-\tau x_{n}^{2}} \mathcal{H}_{\alpha} f\right), n=$ $1,2, \tau=t, s$, for $f \in L^{2}$, we define the $g$-function $G_{N}$ by

$$
\left(\left(G_{N}(f)\right)\left(x_{1}, x_{2}\right)\right)^{2}=\int_{0}^{\infty} \int_{0}^{\infty}\left|t^{N} s^{N} \frac{d^{N}}{d t^{N}} S_{t}^{1} \frac{d^{N}}{d s^{N}} S_{s}^{2} f\right|^{2} \frac{d t}{t} \frac{d s}{s},
$$

where the expression $\frac{d^{N}}{d t^{N}} S_{t}^{1} \frac{d^{N}}{d s^{N}} S_{s}^{2} f$ is understood analogously as in the discrete case. It can be shown that in fact

$$
t^{N} s^{N} \frac{d^{N}}{d t^{N}} S_{t}^{1} \frac{d^{N}}{d s^{N}} S_{s}^{2} f=\mathcal{H}_{\alpha}\left(\left(t x_{1}^{2}\right)^{N}\left(s x_{2}^{2}\right)^{N} e^{-t x_{1}^{2}} e^{-s x_{2}^{2}} \mathcal{H}_{\alpha} f\right) .
$$

Having defined $G_{N}$, we can prove the analogue of Theorem 2.4 (the analogue of the identity of Lemma 3.1 also holds in this case) and Lemma 3.2. This can be done similarly to the discrete case, replacing the sums by integrals (the inner product of the space $L^{2}$ ) and using the fact that the Hankel transform is an isometry on $L^{2}$.

Acknowledgments The author wishes to express his gratitude to Prof. Krzysztof Stempak, for numerous helpful remarks during the preparation of the paper, to Prof. Jacek Dziubański, for suggesting the improvement in the Appendix, and to Prof. Stefano Meda for an instructive conversation.

Open Access This article is distributed under the terms of the Creative Commons Attribution Noncommercial License which permits any noncommercial use, distribution, and reproduction in any medium, provided the original author(s) and source are credited. 


\section{Appendix}

Till the end of the paper, leaving slightly the main line of the reasoning we shall sketch a proof of a multivariate multiplier theorem for the Hankel transform, in which the assumed smoothness threshold $\alpha+\frac{\mathbf{3}}{\mathbf{2}}$ required in the previous section is replaced by $\alpha+\frac{\mathbf{1}}{\mathbf{2}}$. This improvement is achieved by using the dilation/translation structure connected with the Hankel transform setting and appropriately adjusting the proof of the Hörmander multiplier theorem for the Fourier transform from [4, pp. 163-164]. The smoothness requirement will be stated in terms of appropriate Sobolev space norms. For a vector $s$ define the mixed smoothness $L^{2}$ Sobolev space of order $s=\left(s_{1}, \ldots, s_{d}\right)$, by

$$
W^{s}=\left\{\mathcal{F} f \in L^{2}\left(\mathbb{R}^{d}, w_{2 s}(y) d y\right)\right\},
$$

with the norm

$$
\|f\|_{W^{s}}^{2}=\int_{\mathbb{R}^{d}}\left|\mathcal{F} f(y) w_{s}(y)\right|^{2} d y
$$

where $\mathcal{F}$ is the Fourier transform and $w_{s}(y)=\prod_{n=1}^{d}\left(1+y_{n}\right)^{s_{n}}, s \in \mathbb{R}^{d}$. The theorem we obtain is the following.

Theorem 5.3 Letm be a boundedfunction on $(0, \infty)^{d}$. Let $\psi \in C^{\infty}(0, \infty)$, supp $\psi \subseteq$ $[1 / 2,2]$ be such that $\sum_{j \in \mathbb{Z}}\left|\psi\left(2^{-j} \xi\right)\right|^{2}=1, \xi \neq 0$. Let $\Psi\left(y_{1}, \ldots, y_{d}\right)=$ $\psi\left(y_{1}\right) \cdots \psi\left(y_{d}\right)$. If for some $s>\alpha+\frac{1}{2}$

$$
\|m\|_{W_{l o c}^{s}} \equiv \sup _{j \in \mathbb{Z}^{d}}\left\|\Psi m\left(2^{j_{1}} \cdot \ldots, 2^{j_{d}} \cdot\right)\right\|_{W^{s}}<\infty
$$

then the operator $T_{m}$ defined by (5.1) is bounded on all $L^{p}$ spaces, $1<p<\infty$.

As usually, we will prove the theorem only for $d=2$. In higher dimensions the reasoning is analogous. Let $\tau^{y}, y \in \mathbb{R}_{+}^{2}$ be the two-dimensional generalized Hankel translations given by $\tau^{y} f(x)=\tau^{y_{1}} \tau^{y_{2}} f(x)=\tau^{y_{2}} \tau^{y_{1}} f(x)$. Here $\tau^{y_{n}}, n=1,2$, are the one dimensional Hankel translation operators, as defined for instance in [5, p. 3], acting on a function $f$ as a function of $x_{n}, n=1,2$, variable, respectively. The generalized translations can be also expressed as

$$
\tau^{y} f(x)=\int_{\left|x_{1}-y_{1}\right|}^{x_{1}+y_{1}} \int_{\left|x_{2}-y_{2}\right|}^{x_{2}+y_{2}} f\left(z_{1}, z_{2}\right) d W_{x_{1}, y_{1}}\left(z_{1}\right) d W_{x_{2}, y_{2}}\left(z_{2}\right),
$$

with $d W_{x_{1}, y_{1}}\left(z_{1}\right) d W_{x_{2}, y_{2}}\left(z_{2}\right)$ being a probability measure supported in [|x $-x_{1} \mid, x_{1}+$ $\left.y_{1}\right] \times\left[\left|x_{2}-y_{2}\right|, x_{2}+y_{2}\right]$. From the above representation it follows that

$$
\left|\tau^{y} f(x)\right|^{2} \leq \tau^{y}|f|^{2}(x)
$$


It can be also easily checked that $\tau^{y}$ is a contraction on all $L^{p}$ spaces, $1 \leq p \leq \infty$. For any two appropriate functions $f$ and $g$ define their Hankel convolution $f \star g$ by

$$
f \star g(x)=\int_{(0, \infty)^{d}} f(y) \tau^{y} g(x) d v_{\alpha}(y)
$$

It is well known that $\|f \star g\|_{p} \leq\|f\|_{1}\|g\|_{p}$. For a function $f \in L^{1}$ and $\lambda=\left(\lambda_{1}, \lambda_{2}\right)>$ $\mathbf{0}$ define the $L^{1}$ dilation of $f$ by

$$
\left(\delta_{\lambda} f\right)(x)=\lambda^{-2 \alpha-1} f\left(\lambda_{1}^{-1} x_{1}, \lambda_{2}^{-1} x_{2}\right),
$$

where $\lambda^{-2 \alpha-1}=\lambda_{1}^{-2 \alpha_{1}-1} \lambda_{2}^{-2 \alpha_{2}-1}$. Then $\mathcal{H}_{\alpha}\left(\delta_{\lambda} f\right)(x)=\mathcal{H}_{\alpha} f\left(\lambda_{1} x_{1}, \lambda_{2} x_{2}\right)$.

First we need the following comparison lemma.

Lemma 5.4 Let $m_{0}$ be a function supported in $[1 / 2,2]^{2}$. Then

$$
\left\|w_{s} \mathcal{H}_{\alpha} m_{0}\right\|_{2} \lesssim\left\|m_{0}\right\|_{W^{s}}
$$

Proof The one-dimensional version of the above lemma is known, see for instance [5, Lemma 2.9] and references given therein. In our case the lemma follows if we treat two-dimensional Hankel and Fourier transforms as the compositions of the one dimensional transforms acting on separate variables. Then it suffices to apply appropriately the one-dimensional version together with Fubini's theorem.

Our second lemma is the following

Lemma 5.5 Assume that supp $m_{0} \subseteq[1 / 2,2]^{2}$ and that $m_{0} \in W^{s}$ for some $s>\mathbf{0}$. Let

$$
T_{\lambda} f(x)=\mathcal{H}_{\alpha}\left(m_{0}\left(\lambda_{1} \cdot \lambda_{2} \cdot\right) \mathcal{H}_{\alpha} f\right)(x), \quad \lambda=\left(\lambda_{1}, \lambda_{2}\right) \in(0, \infty)^{2} .
$$

Then

$$
\left|T_{\lambda} f(x)\right|^{2} \leq\left\|m_{0}\right\|_{W^{s}}^{2} \int_{(0, \infty)^{2}}|f(y)|^{2} \tau^{y} \delta_{\lambda}\left(w_{-2 s}\right)(x) d v_{\alpha}(y) .
$$

Proof The operator $T_{\lambda} f$ can be represented as a generalized convolution

$$
\begin{aligned}
& T_{\lambda} f(x)=\mathcal{H}_{\alpha}\left(m_{0}\left(\lambda_{1} \cdot, \lambda_{2} \cdot\right)\right) \star f(x)=\int_{(0, \infty)^{2}} \mathcal{H}_{\alpha}\left(m_{0}\left(\lambda_{1} \cdot \lambda_{2} \cdot\right)\right)(y) \tau^{y} f(x) d v_{\alpha}(y) \\
& =\int_{(0, \infty)^{2}} \lambda^{-2 \alpha-1} \mathcal{H}_{\alpha}\left(m_{0}\right)\left(\lambda_{1}^{-1} y_{1}, \lambda_{2}^{-1} y_{2}\right) \tau^{y} f(x) d v_{\alpha}(y)
\end{aligned}
$$


Introducing the factor $w_{s}\left(\lambda_{1}^{-1} y_{1}, \lambda_{2}^{-1} y_{2}\right) \times w_{-s}\left(\lambda_{1}^{-1} y_{1}, \lambda_{2}^{-1} y_{2}\right)$ under the integral sign and applying Schwarz's inequality together with the change of variable $\left(y_{1}, y_{2}\right) \rightarrow$ $\left(\lambda_{1}^{-1} y_{1}, \lambda_{2}^{-1} y_{2}\right)$ we get

$$
\left|T_{\lambda} f(x)\right|^{2} \leq\left\|w_{s} \mathcal{H}_{\alpha} m_{0}\right\|_{2}^{2} \int_{(0, \infty)^{2}}\left|\tau^{y} f(x)\right|^{2} \delta_{\lambda}\left(w_{-2 s}\right)(y) d v_{\alpha}(y)
$$

Now using Lemma 5.4 together with (5.6) and the commutativity of the generalized convolution we get the desired conclusion.

We also need a multivariate Littlewood-Paley theory for the Hankel transform expressed in the following.

Lemma 5.6 Let $\psi$ be a $C^{\infty}$ function, supported in the interval $[1 / 4,4]$, and such that $\sum_{j \in \mathbb{Z}}\left|\psi\left(2^{-j} \xi\right)\right|^{2} \leq C$. Define $S_{j, k}, j, k \in \mathbb{Z}$, by

$$
\mathcal{H}_{\alpha}\left(S_{j, k} f\right)\left(y_{1}, y_{2}\right)=\psi\left(2^{-j} y_{1}\right) \psi\left(2^{-k} y_{2}\right) \mathcal{H}_{\alpha} f\left(y_{1}, y_{2}\right)
$$

Then

$$
\left\|\left(\sum_{j, k \in \mathbb{Z}}\left|S_{j, k} f\right|^{2}\right)^{1 / 2}\right\|_{p} \lesssim\|f\|_{p}
$$

Moreover, if $\sum_{j \in \mathbb{Z}}\left|\psi\left(2^{-j} \xi\right)\right|^{2}=C, \xi \neq 0$, then also

$$
\|f\|_{p} \lesssim\left\|\left(\sum_{j, k \in \mathbb{Z}}\left|S_{j, k} f\right|^{2}\right)^{1 / 2}\right\|_{p}
$$

Proof (sketch). We claim that for $f \in L^{p}\left((0, \infty), d v_{\alpha_{1}}\right)$ and arbitrary $\varepsilon \in\{-1,1\}^{\mathbb{Z}}$

$$
\left\|\sum_{j \in \mathbb{Z}} \varepsilon_{j} S_{j} f\right\|_{L^{p}\left((0, \infty), \nu_{\alpha_{1}}\right)} \leq C\|f\|_{L^{p}\left((0, \infty), \nu_{\alpha_{1}}\right)},
$$

where $\mathcal{H}_{\alpha_{1}}\left(S_{j} f\right)\left(y_{1}\right)=\psi\left(2^{-j} y_{1}\right) \mathcal{H}_{\alpha_{1}} f\left(y_{1}\right)$ and the constant $C$ is independent of $\varepsilon$. If (5.10) holds, then since $S_{j, k}=S_{j} S_{k}=S_{k} S_{j}$, the standard Rademacher function (Khinchin's inequality) trick (see for instance [20, Appendix D.2]) together with Fubini's theorem easily imply the desired conclusion. Coming back to (5.10) it can be shown that the operator $\sum_{j \in \mathbb{Z}} \varepsilon_{j} S_{j} f$ is a Calderón-Zygmund operator with a Calderón-Zygmund constant independent of $\varepsilon$. This can be achieved with the aid of lemmata as for instance [5, Lemma 2.7, 2.8], however we omit the details.

Proof of Theorem 5.3. Let $\widetilde{\psi}$ be another $C^{\infty}(0, \infty)$ function supported in $[1 / 4,4]$ and equal to 1 on $[1 / 2,2]$. Defining

$$
\mathcal{H}_{\alpha}\left(\tilde{S}_{j, k} f\right)\left(y_{1}, y_{2}\right)=\widetilde{\psi}\left(2^{-j} y_{1}\right) \widetilde{\psi}\left(2^{-k} y_{2}\right) \mathcal{H}_{\alpha} f\left(y_{1}, y_{2}\right)
$$


we easily see that $S_{j, k} \tilde{S}_{j, k}=S_{j, k}$ and that the family $\left\{\tilde{S}_{j, k}\right\}$ satisfies inequality (5.8). From (5.9) applied to the family $\left\{S_{j, k}\right\}$

$$
\left\|T_{m} f\right\|_{p} \lesssim\left\|\left(\sum_{j, k \in \mathbb{Z}}\left|S_{j, k} T_{m} f\right|^{2}\right)^{1 / 2}\right\|_{p}=\left\|\left(\sum_{j, k \in \mathbb{Z}}\left|S_{j, k} T_{m} \tilde{S}_{j, k} f\right|^{2}\right)^{1 / 2}\right\|_{p} .
$$

Let $g_{j, k}=\tilde{S}_{j, k} f$. Assume for a moment that $p>2$ and let $1 /(p / 2)^{\prime}+2 / p=1$. Then there exists $u \in L^{(p / 2)^{\prime}}$ with norm 1 such that

$$
\left\|\left(\sum_{j, k \in \mathbb{Z}}\left|S_{j, k} T_{m} g_{j, k}\right|^{2}\right)^{1 / 2}\right\|_{p}=\left(\sum_{j, k \in \mathbb{Z}} \int_{(0, \infty)^{2}}\left|S_{j, k} T_{m} g_{j, k}(x)\right|^{2} u(x) d v_{\alpha}(x)\right)^{1 / 2} .
$$

Since the multiplier associated with $S_{j, k} T_{m}$ is $\Psi\left(2^{-j} y_{1}, 2^{-k} y_{2}\right) m(y)$, from Lemma 5.5 together with Lemma 5.4 it follows that

$$
\begin{aligned}
& \int_{(0, \infty)^{2}}\left|S_{j, k} T_{m} g_{j, k}(x)\right|^{2} u(x) d v_{\alpha}(x) \\
& \quad \leq\|m\|_{W_{\mathrm{loc}}^{s}}^{2} \int_{(0, \infty)^{2}} \int_{(0, \infty)^{2}}\left|g_{j, k}(y)\right|^{2} \tau^{y}\left(\delta_{\lambda}\left(w_{-2 s}\right)\right)(x) d v_{\alpha}(y) u(x) d v_{\alpha}(x) .
\end{aligned}
$$

From the above inequality together with Fubini's theorem we have

$$
\begin{aligned}
& \left(\sum_{j, k \in \mathbb{Z}} \int_{(0, \infty)^{2}}\left|S_{j, k} T_{m} g_{j, k}(x)\right|^{2} u(x) d v_{\alpha}(x)\right)^{1 / 2} \\
& \lesssim\left(\int_{(0, \infty)^{2}} \sum_{j, k \in \mathbb{Z}}\left|g_{j, k}(y)\right|^{2} u \star \delta_{\lambda}\left(w_{-2 s}\right)(y) d v_{\alpha}(y)\right)^{1 / 2} .
\end{aligned}
$$

Since $s>\alpha+\frac{1}{2}, \mathbf{w}_{-2 s} \in \mathbf{L}^{1}$, so that

$\left\|\delta_{\lambda}\left(w_{-2 s}\right) \star u\right\|_{(p / 2)^{\prime}} \leq\left\|\delta_{\lambda}\left(w_{-2 s}\right)\right\|_{1}\|u\|_{(p / 2)^{\prime}}=\left\|\left(w_{-2 s}\right)\right\|_{1}\|u\|_{(p / 2)^{\prime}} \lesssim\|u\|_{(p / 2)^{\prime}}$.

Hence, applying Hölder's inequality we arrive at

$$
\left\|T_{m} f\right\|_{p} \lesssim\left(\sum_{j, k \in \mathbb{Z}}\left|g_{j, k}\right|^{2}\right)^{1 / 2} \|_{p}
$$


Since $g_{j, k}=\tilde{S}_{j, k}$, invoking (5.8) we get the desired conclusion. Now for $1<p<2$ a duality argument completes the proof.

\section{References}

1. Albrecht, D., Franks, E., McIntosh, A.: Holomorphic functional calculi and sums of commuting operators. Bull. Aust. Math. Soc. 58, 291-305 (1998)

2. Cowling, M.G.: Harmonic analysis on semigroups. Ann. Math. 117, 267-283 (1983)

3. Cowling, M.G., Meda, S.: Harmonic analysis and ultracontractivity. Trans. Am. Math. Soc. 340, 733752 (1993)

4. Duoandikoetxea J.: Fourier Analysis. American Mathematical Society, Providence (2001)

5. Dziubański, J., Preisner, M.: Multiplier theorem for Hankel transform on Hardy spaces. Monat. Math. 159, 1-12 (2010)

6. Fraser A.J.: Marcinkiewicz multipliers on the Heisenberg group, $\mathrm{PhD}$ dissertation, Princeton University (1997)

7. Fraser, A.J.: Convolution kernels of $(n+1)$-fold Marcinkiewicz multipliers on the Heisenberg group. Bull. Aust. Math. Soc. 64(3), 353-376 (2001)

8. Fraser, A.J.: An $(n+1)$-fold Marcinkiewicz multiplier theorem on the Heisenberg group. Bull. Aust. Math. Soc. 63(1), 35-58 (2001)

9. García-Cuerva, J., Mauceri, G., Sjögren, P., Torrea, J.L.: Functional calculus for the Ornstein Uhlenbeck operator. J. Funct. Anal. 183, 413-450 (2001)

10. Lebedev, N.N.: Special Functions and their Applications. Dover, New York (1972)

11. Martini A.: Algebras of differential operators on Lie groups and spectral multipliers. http://www.arxiv. org/pdf/1007.1119 (2009)

12. Mauceri, G.: Zonal multipliers on the Heisenberg group. Pacif. J. Math. 95(1), 143-159 (1981)

13. Meda, S.: A general multiplier theorem. Proc. Am. Math. Soc. 110(3), 639-647 (1990)

14. Müller, D., Ricci, F., Stein, E.M.: Marcinkiewicz multipliers and multi-parameter structure on Heisenberg (-type) groups I. Invent. Math. 119(2), 199-233 (1995)

15. Müller, D., Ricci, F., Stein, E.M.: Marcinkiewicz multipliers and multi-parameter structure on Heisenberg (-type) groups. II. Math. Z. 221(2), 267-291 (1996)

16. Nowak, A., Sjögren, P.: Calderón-Zygmund operators related to Jacobi expansions. www.arxiv.org/ abs/1011.3615 (2010)

17. Sikora, A.: Imaginary powers of Laplace operators. Proc. Am. Math. Soc. 129(6), 1745-1754 (2000)

18. Sikora, A.: Multivariate spectral multipliers and analysis of quasielliptic operators on fractals. Indiana Univ. Math. J. 58, 317-334 (2009)

19. Sikora, A.: Sharp pointwise estimates on heat kernels. Q. J. Math. Oxford 47(2), 371-382 (1996)

20. Stein, E.M.: Singular Integrals and Differentiability Properties of Functions. Princeton University Press, Princeton (1971)

21. Stein, E.M.: Topics in Harmonic Analysis Related to the Littlewood-Paley Theory, Ann. of Math Studies, vol. 63. Princeton University Press, Princeton (1970)

22. Thangavelu, S.: Lectures on Hermite and Laguerre expansions, Math. Notes, vol. 42. Princeton University Press, Princeton (1993)

23. Thangavelu, S.: Multipliers for Hermite expansions. Revista Math. Ibero. 3, 1-24 (1987)

24. Veneruso, A.: Marcinkiewicz multipliers on the Heisenberg group. Bull. Aust. Math. Soc. 61(1), 5368 (2000) 